\title{
EDUCACIÓN, JUSTICIA SOCIAL Y MULTICULTURALISMO: \\ TEORÍA Y PRÁCTICA EN EL AULA
}

\author{
Education, social justice and multiculturalism: \\ theory and practice in classrooms
}

\author{
Virginia GUICHOT-REINA \\ Universidad de Sevilla. España. \\ guichot@us.es \\ https://orcid.org/0000-0001-8802-7627
}

Fecha de recepción: 28/04/2020

Fecha de aceptación: 02/08/2020

Fecha de publicación en línea: 01/11/2020

Cómo citar este artículo: Guichot-Reina, V. (2021). Educación, justicia social y multiculturalismo: teoría y práctica en el aula. Teoría de la Educación. Revista Interuniversitaria, 33(1), 173-195. https://doi.org/10.14201/teri.22984

\section{RESUMEN}

Pasados casi cincuenta años desde que apareciera el término «educación multicultural» en Estados Unidos, este trabajo va destinado a mostrar, tras la revisión de la literatura más reciente aparecida en revistas internacionales y editoriales de prestigio, la actual tendencia a vincularla a la consecución de la justicia social, conllevando cuestionamientos acerca del poder y el privilegio y una rebelión contra la opresión que sufren diferentes grupos sociales dentro del sistema hegemónico establecido, e incluimos un ejemplo de implementación en el aula que consideramos muy clarificador. Estos planteamientos impulsan un nuevo tipo de docente que, otorgando un gran valor al diálogo y a la participación del alumnado, le da oportunidades en sus clases para comprometerse con luchas contra la discriminación, unir los valores éticos con acciones políticas, y aplicar ideas de justicia, autonomía y reciprocidad. Concluimos 
haciendo hincapié en la necesidad de desarrollar este enfoque de la educación dirigido a una transformación social en aras de valores como el respeto activo, la libertad, la equidad y la justicia social y que reconoce la diversidad humana como normalidad; más aun teniendo presente el aumento drástico de la desigualdad económica, social y cultural a nivel mundial que suele provocar sentimientos de humillación y de falta de respeto en los grupos desfavorecidos, caldo de cultivo para impedir una convivencia pacífica democrática.

Palabras clave: teoría de la educación; educación multicultural; justicia; formación del profesorado; profesión docente.

\section{ABSTRACT}

After almost fifty years from the appearance of the term "multicultural education" in the United States, this research wants to show the current trend in this area, after the review of the most recent literature published in prestigious international journals and editorials. Multicultural education is going to be associated with social justice. It refers refers not only to a critical interrogation of power and privilege, but also to acts that intentionally disrupt or respond to systemic oppression towards no-hegemonic groups. A very clarifying example of implementation in the classroom is included in this paper. This proposal encourages a new kind of teacher that gives a great value to dialogue and students' participation; offers them opportunities to commit to fighting against discrimination; to linking ethical values with political actions; and to applying ideas of justice, autonomy, and reciprocity. This work concludes emphasizing the need to develop this methodology in education aimed at a social transformation supported by values such as active respect, freedom, equity, and social justice. To summarize education requires a perspective that recognizes human diversity as normal. This is very important, bearing in mind the drastic increase of economic, social, and cultural inequality that tends to provoke feelings of humiliation and disrespect in disadvantaged groups. It is a situation that hinders a peaceful democratic coexistence.

Key words: educational theories; multicultural education; justice; teacher education; teaching occupation.

\section{INTRODUCCIÓN}

Pasados ya casi cincuenta años desde que apareciera el término «educación multicultural» en Estados Unidos, este trabajo va destinado a poner de manifiesto las últimas tendencias dentro de este ámbito, tanto en su concepción como en su puesta en funcionamiento en las aulas, incluidas en el amplio paraguas de las pedagogías críticas, cuyo principal objetivo es un cambio social en profundidad. Bajo estas consideraciones, la educación multicultural va a estar ligada a la justicia social y conllevará cuestionamientos acerca del poder y el privilegio y una rebelión contra la opresión que sufren diferentes grupos sociales dentro del sistema hegemónico establecido. 
En nuestro entorno más próximo, la Unión Europea (UE) existe una preocupación manifiesta por encontrar la mejor forma de abordar la gran diversidad cultural que acoge, una diversidad que se presenta como uno de sus rasgos distintivos. Y ello lo hace sobre la reivindicación, tal y como se consagra en el artículo 2 del Tratado de la UE, de que «la Unión es, ante todo y sobre todo, una Unión de valores, y la educación, la formación y la cultura son cruciales para transmitir y promover valores comunes y desarrollar una comprensión mutua». (Comisión Europea, 2018, p. 1). Hace poco más de dos años, en noviembre de 2017, en la cumbre de Gotemburgo, los dirigentes de la UE eligieron la educación y la cultura como tema principal de debate sobre la base de una contribución de la Comisión titulada "Reforzar la identidad europea mediante la educación y la cultura». (Comisión Europea, 2017). Conscientes de que Europa se enfrenta a retos como los del populismo, la xenofobia, la intolerancia, la discriminación y la desinformación, hacían hincapié en reforzar la dimensión de la educación y la cultura dentro de la Unión, un propósito loable que atiende la educación multicultural ligada a la justicia social que presentamos en este trabajo.

Este artículo se estructura en varios apartados. En primer lugar, hemos contextualizado el origen y los primeros pasos de la denominada «educación multicultural» (principalmente dentro de los Estados Unidos), lo que nos impele a recordar tanto el nacimiento como las consecuencias del modelo neoliberal, hegemónico en la actualidad; sus efectos tanto para la educación en general como para la educación multicultural en particular. Seguidamente, se expone el nuevo enfoque de la educación multicultural ligado a la idea de justicia social, señalando sus principales objetivos, así como el tipo de implementación que requiere. Se descenderá a un ejemplo concreto, puesto en práctica en un college estadounidense por Peggy Shannon, donde quedan reflejados de manera nítida cómo se podría diseñar y poner en funcionamiento un curso de educación multicultural, en este caso en el entorno de la educación superior, que creemos que puede resultar muy clarificador para el profesorado que, a veces, encuentra dificultades para conectar teoría y práctica en el aula (Garreta, 2003).

Resultará entonces mucho más evidente el modelo de formación del profesorado y de perfil docente que consideramos óptimos, que serán presentados una vez mostrados algunos datos de diagnóstico en España sobre la percepción de la educación multicultural por parte de futuros docentes y profesionales de la educación, que revelan las lagunas en la formación dentro de este ámbito. Por último, se establecen una serie de reflexiones, vinculadas la situación sociocultural en la que vivimos, de fuertes desigualdades sociales, económicas y culturales, que nos impulsa a repensar sin demora el mundo en el que deseamos habitar y a comprometernos en su logro. A través de este artículo, pretendemos aportar conocimiento y recursos pedagógicos a los profesionales de la educación que les permitan mejorar su praxis educativa, asumiendo que la multiculturalidad es una realidad en nuestra sociedad que exige que, desde la educación, se ofrezca la respuesta más adecuada 
al alumnado, basándonos en valores esenciales como los de libertad, igualdad y justicia social, pilares de cualquier democracia que se precie.

\section{EL NEOLIBERALISMO Y LA EDUCACIÓN MULTICULTURAL}

El término multicultural education apareció impreso por primera vez en Estados Unidos en 1973 cuando la American Association of Colleges of Teacher Education -la Asociación estadounidense de escuelas de formación del profesorado- realizó el informe titulado No One Model American: A Statement on Multicultural Education (Commission on Multicultural Education, 1973). Este empuje a una nueva forma de entender la educación, caracterizada como "multicultural", nacía en Norteamérica a partir de las demandas del Civil Rights Movement y el desarrollo de estudios étnicos, ya que alentaron propuestas de educación multiétnica; se trataba de movimientos reivindicativos que incluían a grupos que se consideraban a sí mismos en los márgenes de la sociedad, fuera de lo que podríamos llamar los núcleos hegemónicos de poder: población negra, mujeres, personas con distintos tipos de discapacidades, entre otros (Shanon-Baker, 2018; Sleeter, 2018). Su principal meta era conseguir que todos los estudiantes tuvieran las mismas oportunidades de aprendizaje (Banks, 2010), para lo que se requerían acciones desde el nivel micro de las prácticas de clase al nivel macro, esto es, el de las políticas educativas nacionales e internacionales. Claramente, se subrayaba la necesidad de un cambio en el currículo escolar, puesto que este no solo no eliminaba las desigualdades y exclusiones sociales, sino que incluso llegaba a reforzarlas, y, además, silenciaba la voz de los sectores más desfavorecidos de la sociedad.

Conviene recordar que, tras la Segunda Guerra Mundial y el triunfo de los principios económicos keynesianos ligados al Estado de Bienestar — que lograron reducir la brecha de desigualdad social y fueron un apoyo fundamental para la consecución de derechos sociales universales (Judt, 2012)—, un pequeño grupo de economistas conservadores con referentes como Friedrich Hayek ${ }^{1}$ o Milton Friedman, que trabajaban para las élites corporativistas más ricas y poderosas, defendieron los principios neoliberales que las beneficiaban. La tesis básica de esta teoría políticoeconómica sostiene que la sociedad florece y la economía es más robusta cuando se construyen sobre los principios de la libertad individual, la propiedad privada y la competición del mercado. La competitividad, la estandarización, la privatización y un bajo compromiso con la esfera pública constituyen las principales líneas en el plan de acción pensado para los programas sociales, incluido el ámbito educativo (Harvey, 2007). Bajo la teoría neoliberal, la escuela pasa de ser considerada un bien público a un objeto de mercado (Puelles, 2010).

1. La obra de Hayek más conocida, The Road to Serfdom, traducida al castellano como Camino de servidumbre, fue publicada en marzo de 1944 en el Reino Unido y en Estados Unidos. 
El neoliberalismo toma fuerza a fines de los setenta y principios de los ochenta de la mano de la conservadora Margaret Thatcher, primera ministra del Reino Unido entre 1979 y 1990, y del republicano Ronald Reagan, presidente de los Estados Unidos desde 1981 hasta 1989. En un corto espacio de tiempo, extiende su proyecto por todo el mundo y se convierte en el nuevo paradigma global, impulsando una reestructuración social y económica, con consecuencias éticas. Sus tentáculos pronto llegan al mundo educativo y, en los países bajo su influencia, se comenzará a presionar a la educación hacia la competitividad y la privatización con vistas a la ganancia personal (ibídem).

En el terreno educativo, cabe mencionar en los Estados Unidos la publicación de A Nation at Risk, en 1983, por la National Commission on Excellence in Education. Dicho informe promovió la defensa por parte de sectores poderosos de la población de que el principal propósito de las escuelas no debería ser preparar a los niños y jóvenes para una democracia con base en la diversidad (pluralismo social) sino contribuir a recuperar los altos niveles competitivos en el plano internacional de Estados Unidos. Se criticó a las escuelas el no proporcionar a los estudiantes las competencias necesarias para competir en una economía global —obsérvese que ambas palabras tienen una raíz común-. Entre las soluciones aportadas, se propuso fijar estándares explícitos de contenidos para cada una de las disciplinas escolares, así como las pruebas que evaluarían su logro, tarea en la que se enfrascaron los distintos estados estadounidenses. Ello provocó que los docentes se centraran en enseñar conforme a los estándares de contenido, ya que se entendía que era lo que "medía" su buen hacer en las aulas. Esto es, la rendición de cuentas de la escuela hacia la sociedad se enfocó - y se enfoca aún- en que capacitara al alumnado para llegar a esos estándares con los exámenes, normalmente de tipo test o preguntas cerradas, asociados a contenidos tradicionales (Sleeter, 2018). Estas exigencias se extendieron luego a Europa y pronto las encontraríamos en Inglaterra, Francia y otros países de la Unión, nada de extrañar pues responden a la propia globalización del paradigma neoliberal.

Evidentemente, las nuevas directrices neoliberales afectaron a la educación multicultural. Fue redefinida focalizando la atención en adaptar el cómo se enseña, pero dejando frecuentemente atrás el qué se enseña (contenidos) o para qué fines (finalidades), preguntas esenciales en cualquier proyecto educativo. En un sistema sustentado en currículos estandarizados y pruebas «objetivas» para evaluar/controlar los conocimientos del alumnado, poco lugar quedaba, por ejemplo, para discusiones sobre la cultura y la propia construcción del currículum, tan necesarias en una auténtica educación multicultural. Esta se reduce a introducir en los contenidos fragmentos de conocimientos producidos por grupos históricamente marginados. En el campo de la formación del profesorado, en vez de procurar ayudar a los docentes a situar la escuela dentro de una larga historia de luchas en respuesta a opresiones institucionalizadas, se enseña únicamente un conjunto de estrategias para hacer frente a la diversidad cultural pero que nunca pueden actuar como 
auténticas impulsoras de transformaciones sociales profundas (Sleeter, 2018, p. 11). Más que desafiar la inequidad estructural, el centro de atención del multiculturalismo neoliberal consiste en gestionar la diversidad en una sociedad competitiva y altamente desigual (Ibídem, pp. 11-12). En esta línea, afirma Kymlicka (2013, p. 111): "The goal of neoliberal multiculturalism is not a tolerant national citizen who is concerned for the disadvantaged in her own society but a cosmopolitan market actor who can compete effectively across state boundaries».

Con frecuencia, en contextos receptores de fuerte inmigración, la educación multicultural neoliberal se piensa como una forma de solución para la asimilación de nuevos inmigrantes, y como un recurso para construir tolerancia, respeto activo, entre los miembros de la cultura de país de acogida y los inmigrantes (Aguado-Odina, Mata-Benito, y Gil-Jaurena, 2017; Ester Sánchez, 2017); en definitiva, la preocupación se focaliza en ofrecer soluciones destinadas a gestionar el influjo de la diversidad en la sociedad de acogida (por ejemplo, para que no se mine la cohesión social del país). Tal concepción se aproximaría a lo que la UNESCO define como «educación multicultural", concepto que diferencia de "educación intercultural", término que no se usa en el ámbito norteamericano. ${ }^{2}$ Por ejemplo, comenta Antonio T. Ester Sánchez. (2017, pp. 412-413):

La afirmación jurídica del derecho a la educación vendría a entenderse en este sentido como un recurso que llevado a la práctica de forma adecuada posibilita la integración real y efectiva de los miembros pertenecientes a los sectores más débiles de la sociedad, como resulta normalmente ser el caso de la población inmigrante dentro de las sociedades receptoras, facilitando la adhesión a un concepto de sociedad basado en una convivencia pacífica y en una profunda cohesión social (...). Los programas educativos deberán confeccionarse de una manera que resulte acorde con un contenido adecuado a la finalidad de potenciar una eficaz educación democrática, caracterizada por el respeto a la tolerancia y al pluralismo cultural, inspirados en los valores inherentes al propio texto constitucional.

Esta concepción de la educación multicultural se queda bastante alejada de los posicionamientos más cercanos a las pedagogías críticas que abordan directamente el tema del poder, de la hegemonía social, e intentan responder a interrogantes políticos cruciales como: ‘a quiénes corresponde la autoría de la definición de las cuestiones más relevantes y soluciones más idóneas para la mayoría de los mortales?, ¿qué grupo — o grupos- toma las decisiones esenciales sobre la configuración socioeconómica y educativa de nuestra sociedad?, ¿a quiénes beneficia y a quién

2. La educación multicultural usa el aprendizaje de otras culturas para producir aceptación, o al menos tolerancia de esas culturas. La educación intercultural se propone ir más allá de una coexistencia pasiva, para lograr un estilo de vida desarrollado y sostenible en común en sociedades multiculturales a través de la creación de la comprensión, el respeto y el diálogo entre las diferentes culturas. Cfr. UNESCO's Guidelines on Intercultural Education (2006). 
perjudica sus dictámenes?, ¿hacia qué modelo de comunidad nos dirige?, ¿qué valores abrazan?. En esta línea de resistencia al multiculturalismo neoliberal se encuentra, por ejemplo, la denominada Culturally Relevant Pedagogy (CRP), término creado por Gloria Ladson-Billings a comienzos de los noventa del siglo pasado, pedagogía que insta a la acción colectiva basada en la comprensión cultural, las experiencias y las formas de conocer el mundo del estudiantado. ${ }^{3}$ Asimismo, la llamada Culturally Sustaining Pedagogy (CSP), que podemos traducir como «pedagogía culturalmente sostenible», pone el foco de atención en la detección de las injusticias en el sistema, y no en el individuo. Alim y Paris (2017, p. 3) lo explican con estas palabras:

[Culturally sustaining pedagogy] asks us to reimagine schools as sites where diverse, heterogeneous practices are not only valued but sustained. In fundamentally reimagining the purpose of education, CSP demands a critical, emancipatory vision of schooling that reframes the object of critique from our children to oppressive systems.

Aun reconociendo la herencia y valía de la CRP, pretende dar un paso más. En lugar de simplemente aceptar o afirmar los antecedentes de estudiantes de diferentes culturas o conectarse con el conocimiento cultural de los estudiantes, sus experiencias previas y marcos de referencia, como vemos en la CRP, la Culturally Sustaining Pedagogy considera las escuelas como lugares donde las formas culturales diversas se perpetúan y fomentan ("se sostienen»), en vez de erradicarse. Se propone la igualdad entre las comunidades raciales y étnicas y busca garantizar tanto el acceso como las oportunidades de estas. Uno de sus principales objetivos es ayudar al alumnado a criticar y cuestionar las estructuras de poder dominantes en las sociedades (Paris, 2012). Por último, es interesante hacer notar que la manera de abordar la educación multicultural por parte de las pedagogías críticas coincide con ciertas corrientes feministas, como el llamado "feminismo afroamericano" (Fiol-Mata y Chamberlain, 1994), que ofrece los mismos argumentos para explicar por qué las mujeres sufren injusticias y discriminaciones, presentando soluciones similares para lograr la igualdad entre los géneros. Las mujeres serían vistas como un grupo minoritario político y cultural — como los grupos étnicos y raciales- que la élite de hombres ha estado reprimiendo durante- (Abu-Bakker, 2002). Todas estas iniciativas a contracorriente de neoliberalismo se apoyarán en la necesidad de la consecución de justicia social.

3. James Bank (2004) habla de dimensiones de la educación multicultural, que sentaron las bases para avanzar hacia una enseñanza culturalmente relevante: 1. Integración de contenidos (esfuerzo consciente para representar una variedad de culturas en el currículo); 2. Cuestionamiento y análisis crítico del currículo sesgado y previamente aceptado por el alumnado; 3. Fomento de las interacciones interculturales para reducir los prejuicios. 4. Pedagogía equitativa, promotora de un cambio en los enfoques de enseñanza para lograr resultados equitativos para todos los estudiantes y 5 . Adaptación de los métodos de enseñanza para garantizar el éxito de los discentes de todas las culturas. La consecuencia debiera ser una cultura escolar empoderada, con alumnado y profesorado crítico y reflexivo, comprometidos con la justicia social. 


\section{EDUCACIÓN MULTICULTURAL Y JUSTICIA SOCIAL}

En la última década, diferentes autores intentan superar una visión reduccionista de la educación multicultural, de corte neoliberal, que la relaciona únicamente con ciertos contextos como el de la inmigración, el fracaso escolar y la marginalidad (Hinojosa y López, 2018). Proponen un nuevo concepto donde la educación multicultural representa un conjunto de principios, valores y prácticas directamente conectados con la justicia social (Au, 2009; Aguado Odina, 2017; Gorski, 2009; Nieto y Bode, 2012). Por ejemplo, en la definición de Nieto y Bode (2011), se habla de que la educación multicultural acoge y afirma los fines y valores democráticos, el pluralismo cultural, la justicia social, así como el desarrollo intelectual, personal y social de los estudiantes. Y siguiendo a Banks (2013), debe asegurar la igualdad de todos los estudiantes en la educación sean cuales sean sus diferencias individuales (lenguaje, religión, raza, edad, género, nivel económico, clase social). Asimismo, la National Association for Multicultural Education (NAME), en un documento de 2014, señalan seis puntos clave de la educación multicultural:

a) Las escuelas juegan un rol significativo en mejorar las actitudes y los valores de los estudiantes que requiere una sociedad democrática.

b) Adopta diferentes valores y pluralismo cultural.

c) Promueve el desarrollo de la justicia social y prohíbe la discriminación de cualquier clase en escuelas y en la sociedad.

d) Todas las actividades y políticas implementadas en la escuela tienen un efecto positivo sobre el conocimiento y niveles de destreza de los estudiantes.

e) Los estudiantes toman parte activa en todas las instituciones y organizaciones con igualdad.

f) Es un proceso que anima a profesores y estudiantes a analizar críticamente el mundo de hoy donde prevalece el poder y la dominación.

Ukpokodu (2016) define las teorías y prácticas pedagógicas que incluyen el punto de vista de la justicia social como una pedagogía que supone la integración de cuestiones sobre justicia social de forma intencional, explícita y sistemática. Implica no solo un cuestionamiento crítico sobre quién detenta el poder y goza de los privilegios y quiénes padecen discriminaciones, sino también acciones, prácticas, que luchan contra la opresión sistémica (Salazar y Ríos, 2016). A nuestro entender, se acerca al planteamiento freireano que busca la desaparición de la dicotomía opresor-oprimido a partir de un modelo que incluye la concientización pero que no termina ahí, puesto que necesita del paso imprescindible de la acción reflexiva, la praxis, para cobrar pleno sentido (Freire, 1984). Una visión freireana que, en 
nuestra opinión, empapa las propuestas que conectan educación multicultural con justicia social: cuestionamiento de la realidad y análisis de los mecanismos de opresión y de las relaciones de poder por toda la comunidad educativa, partiendo de dos premisas: a) un aprendizaje de todos entre todos y b) la construcción social del conocimiento.

Siguiendo a Penny Shannon-Baker (2018), una praxis de educación multicultural empodera a los participantes, construye una auténtica comunidad entre profesorado y alumnado, comparte el poder y analiza críticamente tanto las estructuras institucionales como las prácticas personales que mantienen sistemas opresivos. Anima a preguntar cómo se construye y se comunica el conocimiento, cómo interactuamos y apoyamos a los otros, especialmente con los más diferentes a nosotros mismos; y cómo la diversidad se representa o se elimina (Banks, 2010; Herrera, Holmes, \& Kavimandan, 2012). Esta praxis incluye la promoción de la justicia cognitiva; esto es, no hay justicia social sin el derecho efectivo a la igualdad para vivir desde las posibilidades mentales que nos proporciona nuestra cultura (Sousa, 2012). Supone, pues, la aceptación de varias formas de conocimiento, a través de la integración de caminos no hegemónicos de conocer (Muchenje, 2017).

La ruptura de la dicotomía profesor/a versus alumno-a, nos lleva a hablar, al modo freireano, de educador-educando y de educando-educador (Freire, 2001) que construyen conjuntamente la clase, los conocimientos. Ello altera las nociones de poder, impulsando una concepción de la relación educativa mucho más horizontal. Se busca crear colectivamente, a ser posible por toda la comunidad educativa, espacios de aprendizaje compartidos donde ser reflexiona críticamente sobre prejuicios, privilegios y poder (Howard, 2003; Shannon-Baker y Talbot, 2016). Una pedagogía basada en la justicia social permite a los estudiantes construir y usar el conocimiento para llegar a ser agentes de cambio tanto a nivel individual como social (Oakes, Lipton, Anderson, y Stillman, 2016).

Esta concepción de la educación multicultural ligada a la justicia social queda más clara mostrando un ejemplo del diseño y la puesta en práctica de un curso de educación multicultural que abraza esta filosofía de la educación. ${ }^{4}$ Su autora e implementadora fue Penny Shannon-Baker (2018) en un college de élite estadounidense, una institución de educación superior, exclusivamente femenina. Inicialmente el curso solo fue pensado para futuras profesoras, pero al final se abrió para todas las estudiantes del college y de otras instituciones vecinas, asistiendo en total

4. Queremos enfatizar que la justicia social no puede ser entendida como el diseño de un programa determinado y su aplicación en un contexto dado. Se trata de uno de los fines últimos a los que debe dirigirse la educación. Resultan clarificadoras al respecto las palabras de Teresa Aguado que define la educación intercultural como un enfoque teórico que entiende: «la educación como procesos socioculturales que contribuyen a formar personas libres y autónomas; capaces de pensamiento crítico y participantes activos en la sociedad. Se reconoce la diversidad humana como normalidad (...). El fin último es la igualdad y la justicia social». (Aguado, 2017, p. 22). 
25 personas. En cuanto a su composición, era variada en términos de edad, curso académico, especialidad (educación, psicología, sociología), raza/etnia (quince se autoidentificaban como estudiantes de color), clase social (algunos eran de clase trabajadora y primera generación que disfrutaba de educación superior en su familia) y diversidad sexual (varias eran componentes del colectivo LGBTQ). Su duración fue de un semestre con clases dos veces por semana. Las tareas para las estudiantes incluían diez diarios personales semanales, notas de observación realizadas en trabajo de campo durante 16 horas, un proyecto de investigación llamado Showcase Project (Proyecto de Exposición) y un portfolio de reflexiones finales. La propia Shannon, en su papel de docente en el curso, se planteó aprender a través de la reflexión en la acción (reflexión sobre el trabajo mientras se efectúa) y la reflexión sobre la acción (reflexión hecha retrospectivamente) (Schön, 1987). Utilizó como fuente para los datos: diarios del profesor/a semanales, materiales de lectura, notas del diseño del curso, programa del curso, programaciones y trabajos de las estudiantes, entre otros. Cuando el curso se completó, compiló los datos y usó las siguientes preguntas para guiar su análisis:

- ¿Cuándo mi práctica ha materializado una praxis de educación multicultural? ¿Qué actividades específicas, lecturas, tópicos y formatos de debate conllevó?

- ¿Cuándo mi práctica no ha encarnado una praxis de educación multicultural? ¿Qué falló y por qué?

- ¿Qué cambios podrían hacerse en el futuro y por qué? ¿Qué revelan los descubrimientos hechos sobre la escuela y sobre la educación multicultural? ¿De qué forma los descubrimientos consideran cuestiones más amplias sobre la educación para la justicia social?

Su curso fue organizado en torno a una serie de cuestiones (topics) trabajadas mediante distintas estrategias que se muestran en la siguiente tabla:

TABLA 1

Temas y estrategias para el aula en el curso de educación multicultural

\begin{tabular}{|c|c|}
\hline TEMAS (topics) & ESTRATEGIAS PARA EL AULA \\
\hline $\begin{array}{l}\text { Definiendo y } \\
\text { enmarcando } \\
\text { la educación } \\
\text { multicultural dentro } \\
\text { de sus contextos } \\
\text { históricos y } \\
\text { contemporáneos }\end{array}$ & $\begin{array}{l}\text { - Investigar la historia del concepto de «educación multicultural». } \\
\text { - Discutir sobre actuales movimientos promotores de justicia } \\
\text { social. } \\
\text { - Analizar versiones «oficiales» (safe) de la Historia versus } \\
\text { versiones más críticas; diseñar más lecciones críticas. } \\
\text { - Utilizar los actuales medios de comunicación y tecnología } \\
\text { como un medio para las tareas y las discusiones. }\end{array}$ \\
\hline
\end{tabular}




\begin{tabular}{|c|c|}
\hline TEMAS (topics) & ESTRATEGIAS PARA EL AULA \\
\hline $\begin{array}{c}\text { Conectar las teorías a } \\
\text { experiencias vividas } \\
\text { y viceversa }\end{array}$ & $\begin{array}{l}\text { - Leer y discutir testimonios con empatía crítica y responsabilidad } \\
\text { personal. } \\
\text { - Empezar por cuestiones locales/regionales. } \\
\text { - Utilizar recursos multimedia, ej. filmes, presentaciones } \\
\text { interactivas y cómics. } \\
\text { - Emparejar lecturas de cultura popular/divulgativa con escritos } \\
\text { teóricos. } \\
\text { - Animar a los/las estudiantes a compartir mediante compartir } \\
\text { sus propias historias. } \\
\text { - Defender una aproximación anti-turismo de voluntariado en } \\
\text { las experiencias de campo multiculturales. }\end{array}$ \\
\hline $\begin{array}{c}\text { Enseñar y modelar } \\
\text { una praxis } \\
\text { de educación } \\
\text { multicultural }\end{array}$ & $\begin{array}{l}\text { - Compartir prácticas de enseñanza, decisiones y formas de } \\
\text { razonar con estudiantes cuando sea posible. } \\
\text { - Asignar proyectos que fomenten la autonomía de los/las } \\
\text { estudiantes. } \\
\text { - Diseñar la rúbrica de las tareas como clase (decisiones } \\
\text { compartidas). } \\
\text { - Facilitar el feedback entre iguales formando grupos de } \\
\text { estudiantes de forma intencional. Proporcionar una estructura } \\
\text { poca definida para guiar el feedback. } \\
\text { - Reconocer de manera pública a la clase errores y prácticas que } \\
\text { trastocaron la praxis. } \\
\text { - Involucrar en reflexiones críticas simultáneas y retrospectivas; } \\
\text { compartir mientras sea posible. }\end{array}$ \\
\hline
\end{tabular}

Fuente: Versión española traducida por Virginia Guichot a partir de Shannon (2018, p. 52)

Shannon recomienda evitar el error de muchos cursos de educación multicultural que se imparten en Estados Unidos, organizados temáticamente por variables/ pertenencias que forman parte de la identidad, como raza o clase socioeconómica, sin establecer conexiones entre ellas. Es necesario tener en cuenta la conocida como "teoría de la interseccionalidad». 5 Por ejemplo, dentro del colectivo «inmigrantes", no es lo mismo ser hombre blanco europeo con buenos medios económicos, que mujer blanca europea pobre. Otra equivocación es separarlos de movimientos

5. El enfoque conocido como «interseccionalidad» fue acuñado en 1989 por Kimberlé Williams Crenshaw, actual profesora de Columbia donde dirige el Centro sobre Interseccionalidad y Estudios Sociales. Collins y Bilge definen la interseccionalidad como una herramienta para analizar cómo las relaciones de poder se entrelazan y construyen mutuamente. La raza, la clase, el sexo, la sexualidad, la dis/capacidad, la etnia, la nacionalidad, la edad, son categorías de análisis, términos que hacen referencia a importantes divisiones sociales, pero también son categorías que adquieren significado a raíz de las relaciones de poder del racismo, el sexismo, el heteropatriarcado, y la explotación de clases (Collins y Bilge, 2016, p. 7). 
sociales actuales como Black Lives Matter, Native Lives Matter, o el Undocumented Student Movement.

Es necesario cuestionar cómo se cuentan, cómo se narran, ciertos acontecimientos relacionados con algún colectivo que ha sufrido marginación, al querer desarrollar una educación multicultural. Puede ocurrir que se esté favoreciendo el statu quo establecido y por tanto la visión histórica que interesa a los grupos hegemónicos, en lo que Shannon denomina la clásica versión de «héroes y festividades». Un caso clarificador es cómo se presenta en colegios e institutos el caso de Rosa Parks reclamando quedarse en su asiento en el autobús y no cederlo a un joven varón blanco; a veces, tal como se trabaja en algunos centros educativos, podría parecer que la principal razón era que estaba cansada y no que había sido formada para realizar actos reivindicativos de no-violencia a través de grupos de activistas organizados pro-derechos civiles. ${ }^{6}$ Shannon, por ejemplo, como profesora del curso de educación multicultural, animó al alumnado a que comprobase qué versión "oficial» (en libros de texto, etc.) se suele dar de los hechos, según las edades; a que criticasen las implicaciones de enseñar ese tipo de narrativa e identificasen fuentes críticas para trabajar con su futuro alumnado dependiendo de la edad. Dicha información fue luego compartida en el sitio web de la clase.

Algo que creemos especialmente atractivo de esta experiencia es la idea de fomentar que el estudiantado vaya compartiendo los aprendizajes que van haciendo a través de sus redes sociales, como Twitter o Facebook, una magnífica forma de que éstos se divulguen y lleguen a muchas más personas. Además, es una estupenda manera de lograr que sigan hablando de ellos fuera de la clase debido a que los y las estudiantes van respondiendo a los comentarios que les hacen por las redes.

Otra práctica utilizada en este curso fueron las narrativas sobre experiencias vividas. Ellas permiten no solo empatizar con la persona de la historia, sino también demanda que nos interroguemos sobre nuestra responsabilidad en las experiencias de esa persona (Boler, 1997). Se trata de tener que aceptar la complicidad y responsabilidad personal en determinadas situaciones para tornar nuestro conocimiento y empatía en acción.

En una línea similar, dentro del curso se hacían prácticas que buscaban partir de las propias vidas de los implicados para comprender teorías y prácticas relacionados con la educación multicultural. Por ejemplo, se pidió a las alumnas que escribieran sobre algo que ellas hubieran logrado tras grandes esfuerzos. Entonces, analizaron estas narrativas relacionándolas con el conocido como «el sueño americano» 7

6. Rosa Parks, en 1950, se había unido al movimiento de derechos civiles y se empleó como secretaria de la National Association for the Advancement of Colored People en Montgomery. El incidente del autobús pasó el 1 de diciembre de 1955, cinco años después.

7. Este ideal, clave en el imaginario nacional estadounidense, abre las puertas a un futuro esplendoroso disponible para quien lo desee, gracias a la movilidad social. Ahora bien, según datos recientes de la OCDE (2018), Estados Unidos es uno de los países desarrollados donde la movilidad social es 
—que viene a decir: «si te esfuerzas lo suficiente, al margen de tus raíces familiares y de tus orígenes socioeconómicos, podrás llegar allí donde te lo propongas»—, y con el neoliberalismo con su énfasis sistemático en la objetividad, la meritocracia y el individualismo (Augoustinos, Tuffin, y Every, 2005). En clase, hablaron sobre cómo esos discursos centrados en el individuo, la suerte y la meritocracia, borraban todo lo referido al acceso estructural al privilegio, la importancia de la familia y el impacto de la ayuda de los otros. El alumnado analizó entonces sus propias narrativas y añadió las personas o estructuras que les habían ayudado. La semana siguiente, Shannon compartió también sus experiencias en torno a la posesión del grado de Doctora y las ventajas que recibió por parte de instituciones e individuos. El resultado fue doble: romper la retórica del éxito solo dependiente del individuo propia de los Estados Unidos y practicar el desvelamiento de privilegios o desventajas para el logro de un objetivo escribiendo las propias historias personales de una forma que el alumnado podía reconocer tanto los privilegios personales como los estructurales. De esta manera, se fue mucho más allá del primer acercamiento mediante la lectura de testimonios al preguntarse cada uno de los estudiantes cómo estaba implicado en sistemas de privilegios.

Tras ocho semanas de trabajo de campo en espacios multiculturales (escuelas, actividades extraescolares...) donde el alumnado debía conectar la teoría con la práctica, el curso finalizaba con una exposición por grupos de un proyecto multimodal (Showcase Project). Aquí la persona docente proporcionaba un conjunto de preguntas estructuradas que les ayudaran a trabajar en la cuestión elegida para que el estudiantado no se sintiera desorientado, que era una queja común en el alumnado. Por ejemplo, se insistía en la necesidad de considerar tres interrogantes clave: a qué audiencia va dirigido el proyecto, cuál es el mensaje y cuál es el mejor medio para comunicar ese mensaje a la audiencia. Entre todos, profesora y estudiantes, crearon la rúbrica de evaluación, una magnífica forma de asegurarse de que todo el mundo estaba de acuerdo y comprendía qué se valoraba.

Para realizar el proyecto, se empleaba la segunda mitad del semestre, incluyendo reuniones individuales con la docente, tiempo de trabajo en clase, talleres con iguales, y dos días finales en los que todos los grupos compartían sus proyectos con la clase. Un ejemplo de proyecto fue la creación de una página web sobre cómo hablar a adolescentes de la escuela secundaria sobre raza. Los proyectos eran multimodales porque Shannon se dio cuenta de que el trabajo de investigación tradicional estaba actuando como una barrera para visualizar cómo se podría materializar la educación multicultural en la práctica diaria. Por ejemplo, a través de la creación de un

más limitada. Es decir, si una persona nace en una familia pobre, tiene más difícil escapar de la pobreza cuando sea adulto. La OCDE lo ha calculado tomando como referencia el $25 \%$ más rico de la población de cada país: en Estados Unidos, menos del 10 \% de la población más próspera cuenta con orígenes humildes, pobres. Cfr. OECD (2018). 
vídeo o de un libro infantil, los estudiantes tuvieron que comprender no solo su contenido sino también cómo adaptarlo a la audiencia a quien iba dirigido y cuál era la mejor forma de comunicarlo.

Creemos que esta experiencia es muy clarificadora sobre la manera de relacionar el trabajo en educación multicultural con el tema de la justicia social, y nos da muchas pistas y sugerencias acerca de cómo podríamos diseñar un curso para futuros docentes a nivel universitario. ${ }^{8}$

\section{EL PROFESORADO: REALIDAD Y NUEVOS HORIZONTES}

Cualquier curso o programa de educación multicultural solo puede tener éxito si cuenta con un profesorado preparado para impartirlo, lo que implica, aparte de una serie de conocimientos profesionales, contar con una serie de actitudes y valores (Freire, 2001). Como cabría esperar, el pensamiento del profesorado acerca de la diversidad cultural se refleja en el diseño de sus clases, en sus actividades en el aula, y en sus expectativas sobre los y las estudiantes y su desarrollo académico y personal. Estudios recientes (OECD, 2009; Dedeoglu y Lamme, 2011; Yang y Montgomery, 2011) verifican esta correspondencia compleja entre conocimiento, creencias, y prácticas de enseñanza en el aula. Desgraciadamente, numerosos estudios critican el bajo nivel de compromiso hacia la diversidad cultural y la resistencia a incentivar más prácticas de formación inclusiva ligadas a una aproximación intercultural por parte de los y las docentes (Leiva, 2008; Nelson y Guerra, 2013). A pesar de que se presta gran relevancia a la educación intercultural/multicultural desde el punto de vista teórico (Grant y Gibson, 2011; Hunter, Pearson y Gutiérrez, 2015) y por organizaciones internacionales (European Commission, 2010; OECD, 2010), no parece que se esté haciendo un gran esfuerzo para su logro en la formación del profesorado, lo que conlleva prácticas inadecuadas o de gran pobreza (Bhopala y Rhamie, 2014; Essomba, 2010; Moloney y Saltmarsh, 2016).

Hinojosa y López (2018) realizaron en España una investigación con 1464 participantes dentro del profesorado en formación inicial a través de cuestionarios. Es interesante revisar algunas de sus conclusiones. Quizá la más destacada, dado el objeto de nuestro trabajo, es la limitada y restrictiva visión de la diversidad cultural que tienen la mayoría de estos futuros docentes, que se une a su tendencia a enlazar diversidad cultural con inmigración, fracaso escolar y contextos marginales. En cambio, la nueva concepción de educación multicultural que hemos presentado remarca la idea de que la diversidad cultural ha de pensarse como un rasgo

8. En el contexto español, el libro Educación intercultural (2017) coordinado por Teresa Aguado y Patricia Mata ofrece múltiples recursos y actividades entendiendo la educación intercultural como «una metáfora que nos permite comprender lo que pensamos, decimos y hacemos en relación con la diversidad cultural desde la asunción de un compromiso por la equidad y la justicia social». (p. 21). 
consustancial a la sociedad actual que no está limitado a escenarios específicos o marginales. Asimismo, estos futuros maestros y maestras afirman ser conscientes de la naturaleza deficiente de su formación en este campo y reclaman que se le preste más atención, toda una llamada de atención tanto a nivel macro, esto es, a las políticas educativas desarrolladas, como al nivel micro, vinculado a la realidad del aula. Un tercer resultado refiere al salto de la teoría a la práctica. Aunque los sistemas de creencias de los participantes tienden a converger cuando se refieren a aspectos superficiales y generales de la diversidad cultural, la convergencia es escasa en el campo de transferencia de los principios generales de la diversidad cultural a la práctica de enseñanza, al currículum y a situaciones específicas. Esta circunstancia implica — comentan Hinojosa y López (2018) — una falta de reflexión sobre los efectos de adoptar esos principios en la práctica de enseñanza y una pobre enseñanza práctica (en los centros de formación del profesorado). El alumnado participante suele tener una actitud relativista hacia la diversidad cultural y una falta de pensamiento crítico, revelándose por afirmaciones como la de que "todas las formas culturales existentes deben ser aceptadas", algo que parece afectar a más de un campo en las Universidades (Giroux, 2019). En definitiva, existen significativas debilidades epistemológicas y prácticas en el terreno de la educación multicultural en la formación del profesorado inicial (Hinojosa y López, 2018, pp. 88-89).

A partir de estas conclusiones, es importante observar la necesidad de replantear la formación del profesorado en el campo de la educación intercultural, tal como se la denomina en el ámbito europeo. Una autoridad en la materia es Gloshanda Lawyer (2018), quien proporciona algunas recomendaciones para los docentes que impartan cursos tanto a los futuros profesores como a aquellos que ya están ejerciendo como tales.

La primera recomendación consiste en reconocer la tensión entre los estudiantes que viven el contenido del curso y aquellos que están aprendiendo desde la perspectiva del outsider. Es decir, el profesorado debe darse cuenta de que hay estudiantes que están dentro de los grupos no hegemónicos, que se han visto generalmente envueltos en casos de discriminación y de ausencia de poder, y que otros no, a la hora de trabajar con ellos. En este sentido, creemos fundamental la necesidad de ser empáticos, de saber ponerse en el lugar del otro, de ser sensibles a sus experiencias, en numerosos casos de sufrimiento y ansiedad por ser marginados.

Una segunda propuesta se encamina hacia una función esencial que debe tener el sistema educativo que es la de crear individuos críticos puesto que Lawyer aconseja al profesorado proporcionar dirección a los estudiantes para saber dónde hacer más preguntas. Se trata de impulsar al alumnado a que se cuestione la realidad, a que no naturalice las situaciones, a que se interrogue sobre las causas que provocan los hechos, a que se pregunte a quién favorece y a quién perjudica determinadas políticas establecidas..., algo que precisamente advierte Giroux (2019) que se está perdiendo cada vez más en las universidades. 
El tercer consejo habla de acercarse al contexto de los discentes, desafiando las faltas de equidad institucionales que tantas veces existen, a través del uso de recursos (académicos y no académicos) provenientes de perspectivas de las minorías o de gente marginada: dar voz a los que no tienen voz; considerar la apropiación del conocimiento que hacen grupos no hegemónicos... Un ejemplo lo podemos observar en los mensajes de los grafitis o en las letras de determinados estilos musicales propios de población marginal (Gramigna, 2007).

Varias recomendaciones dirigen la atención a la importancia de dar testimonio con nuestras acciones de aquello que predicamos. En clase, el o la docente debe interrumpir cualquier conducta de opresión que detecte y ha de convertirla en un momento para tomar conciencia de ella con miras a eliminarla, es decir, transformarla en una situación de aprendizaje para el estudiantado. Una finalidad parecida tiene la exploración de las intersecciones de la opresión con marcadores de identidad (ejemplo, ser una persona de color y ser mujer: raza y sexo) para evitarla cuando se manifieste en el curso. Asimismo, el profesorado tiene que hacer activismo encaminado a la justicia social en sus clases, porque será una forma ideal de demostrar al alumnado su auténtico compromiso con el cambio hacia un mundo más equitativo, libre y humano.

La nueva concepción de la educación multicultural y los consejos sobre su impartición nos permiten crear un bosquejo de perfil ideal que debería poseer el profesorado. Enlazando con el marco conceptual y práctico que hemos presentado, el personal docente tendría que pensar la educación intercultural como un enfoque teórico que entiende la educación como procesos socioculturales que fomentan la creación de personas libres, autónomas, críticas y participativas en una sociedad donde la diversidad humana debiera verse como normalidad y cuyo fin último sería la igualdad y la justicia social (Aguado, 2017, p. 22). Un concepto de educación que, por consiguiente, supone un compromiso de transformación social. En este perfil ideal, hemos señalado ocho rasgos que nos parecen esenciales, construidos mediante las aportaciones de distintos autores y autoras, pero, sin duda, está abierto a nuevas contribuciones:

a) Tener una valoración positiva de la diversidad cultural (Torres, 2008) y entenderla como un incentivo que nos impulsa a repensar nuestras creencias, nuestras formas de enfrentarnos a los problemas, a la vida misma. Como dice Pinedo (2013): "Quedarnos apegados a nuestras raíces solo por el hecho de sentirnos a gusto entre los que piensan como nosotros puede ser un signo de estancamiento mental, pues donde todo el mundo piensa igual, nadie en realidad está pensando mucho». (p. 189). Quien ejerce la docencia debe ser consciente de su propio capital cultural, de su adscripción social, de su sexo y de su pertenencia étnica, consciente de que todo ello influye y sesga su percepción del mundo, su pensamiento y su acción. 
b) Reconocer culturas silenciadas y hacerlas visibles (Torres, 2008). El profesorado ha de ser consciente de la parcialidad del currículo escolar de cómo penaliza, oculta, y a veces descalifica las experiencias, conocimientos y actitudes de ciertos grupos sociales y debe responder a ello eliminando esas discriminaciones. Por ejemplo, ante la ausencia de personajes femeninos ligados a la ciencia o al arte en los libros de texto, buscar casos y además investigar sobre sus vidas para descubrir los obstáculos que sufrieron para desarrollar la actividad.

c) Defender la justicia social como un camino que garantiza la equidad; prestar atención a las necesidades de los y las estudiantes sin olvidar sus desigualdades iniciales y las desventajas procedentes de factores socioeconómicos (Cochran-Smith, 2010). No hay nada más injusto que dar lo mismo a los que parten de situaciones distintas.

d) Tomar decisiones curriculares colectivas, consensuadas y dialogadas (Caetano, Freire y Machado, 2020; Hinojosa y López, 2018; Sleeter, 2018,). El diálogo comienza cuando cada parte es reconocida y legitimada; necesita de la aceptación de tu interlocutor como un otro legítimo. Se trata de ponernos de acuerdo sobre qué es justo y qué es injusto para una comunidad de personas diferentes que se unen en un proyecto común. A través del diálogo con el otro distinto tiene lugar el encuentro entre culturas que enriquece, que posibilita el intercambio y la construcción de las propias bases para la comunicación. Señala Ruíz de Lobera (2004) que, desde la perspectiva intercultural, cuanta más responsabilidad, es decir, cuanta más capacidad se dé a la persona en la toma de decisiones, más verdaderamente formativa y participativa es la actividad.

e) Fortalecer y practicar la solidaridad y la cooperación como alternativas a la competición y al individualismo para la consecución de la cohesión social necesaria para la construcción de un proyecto común democrático (Hinojosa y López, 2018; Pinedo, 2013). Indica Bartolomé Pina (2004) que el valor que debemos desarrollar para la formación del sentimiento de pertenencia a una comunidad o sociedad es el de la solidaridad inclusiva, descubriendo que la causa de quien vive a mi lado es mi propia causa, aunque sea diferente de mí, y estar dispuesto por ello a actuar a favor de su causa como si fuera la mía propia.

f) Tomar conciencia de que como docente es un intelectual, no solo un técnico (Giroux, 1990), que debe tener compromiso público, responsabilidad ética, y conciencia de su misión en construir un futuro mejor, más equitativo y respetuoso con la diversidad. En definitiva, debe tener cualidades como compromiso ético y social, imaginación y preocupación por los demás (Hargreaves y Shirley, 2012; Tarozzi, 2014). En este sentido, señala Gay 
(1995, p. 179) que debe dar oportunidades en sus clases a los y las estudiantes para comprometerse con luchas contra la opresión, unir los valores éticos con acciones políticas, y aplicar ideas de justicia, autonomía y reciprocidad. Asimismo, ha de comprender que muchas causas de opresión se interrelacionan, y así debe trabajarlas en clase.

g) Ser humilde, esto es, reconocer nuestras propias faltas y errores en la clase y discutirlos con la clase como un medio de romper las dinámicas de poder profesor-estudiante (Shannon, 2018, p. 57). Es la conciencia de saber que nadie lo sabe todo, y nadie lo ignora todo: aprendemos los unos de los otros (Freire, 2001).

h) Comprender la enseñanza como una actividad problemática que debe estar basada en el análisis de sus teorías implícitas y de una reflexión crítica sobre sus creencias. Carbonell (1995) hablaba de adoptar "la actitud del ignorante» que permite cuestionar nuestros propios esquemas teóricos de análisis de la realidad y escuchar al otro con atención si queremos conocerlo de verdad, compartir con él proyectos, ilusiones y situaciones de la vida cotidiana.

\section{REFLEXIONES FINALES}

Señala Fukuyama en su reciente libro Identidad (2019) que las democracias liberales contemporáneas no han resuelto satisfactoriamente el problema de thymós, que es la parte del alma que anhela el reconocimiento de la dignidad. Prometen y en gran medida brindan un grado mínimo de igual respeto, encarnado en los derechos individuales, el Estado de derecho y el sufragio, pero ello no garantiza que las personas sean igualmente respetadas en la práctica, particularmente los miembros de grupos con un historial de marginación. Si a ello unimos el aumento drástico de las desigualdades a nivel económico, particularmente en las democracias desarrolladas (Milanovic, 2018), tenemos el caldo de cultivo perfecto para originar fuertes tensiones sociales y conflictos violentos, que pueden llegar a desembocar incluso en guerras civiles.

Este inquietante cuadro nos conmina a pensar cómo podemos actuar para el logro de un mundo más justo para todos y todas, que se tome en serio el respeto a la dignidad humana como principal valor y, por tanto, como principio que defina las políticas sociales, económicas y culturales adoptadas. Como profesionales de la educación, nuestro deber es proponer una concepción de educación —que llevará asociada determinadas prácticas - destinada al logro de los valores democráticos que garantizan ese respeto a la dignidad, de modo que nadie se sienta menospreciado, ninguneado o humillado, y todos, sin excepción, se vean comprometidos en la tarea irrenunciable de construcción de un mundo mejor para el conjunto de la ciudadanía, de ese bienestar común tan necesario. Creemos que la idea de 
educación intercultural que hemos descrito en este artículo, que se incluye en un proyecto ético y político, cumple plenamente este objetivo.

Se ha defendido que fomentar los procesos de educación intercultural va más allá de conocer otras culturas o participar en sus expresiones folclóricas (comidas típicas, bailes, etc), hablar otra lengua, o adoptar una visión ingenua de la diversidad cultural (Leite, 2005; Tarozzi, 2014). Debe significar un posicionamiento que contempla individuos y contextos sociopolíticos y orienta la enseñanza hacia la justicia social y el pensamiento crítico (Cochran-Smith, 2010). Ha de suponer un espacio de reflexión para discutir y formular puntos de vista éticamente sostenibles que contribuyan a una mejor convivencia (Pinedo, 2013). Una buena educación intercultural favorece, además, como señala Bhikhu Parhek (2005), el desarrollo de capacidades humanas tan valiosas como la curiosidad intelectual, la capacidad de autocrítica, la habilidad para sopesar argumentos y evidencias y formar juicios independientes.

A modo personal, queremos comentar que, desde nuestros inicios como docente, nos planteamos vincular nuestro trabajo con la justicia social, con proporcionar conciencia y alternativas para transformar la realidad en una línea más humanizadora. Una justicia que, en el campo educativo, debe contemplar dimensiones como una igualdad de acceso y oportunidades para todo el alumnado, pero también, de manera esencial, el reconocimiento de la diversidad, esto es, el respeto que merece la dignidad que cada uno alberga, y la participación activa en decisiones curriculares significativas (mayor o menor según la edad) (Friant y Sánchez-Santamaría, 2018). Conocimos a personas que empezaron como nosotras, pero que han ido cayendo en un pesimismo paralizante, que no por comprensible, resulta muy negativo porque contribuye a mantener el statu quo. Por eso, a través de este trabajo, nos gustaría animar al profesorado a que mantenga en todo momento la esperanza, incluso en las peores circunstancias, y que siempre piense desde su posición vital qué es lo que puede hacer para mejorar la sociedad, conscientes de que no basta con querer, sino que hay que "saber querer" (Freire, 2001, p. 78). Solo con la educación no se cambia un mundo lleno de injusticias, pero, sin ella, no se puede hacer. Y entre otras cosas, debemos buscar alternativas para un sistema neoliberal que, por ser infinitamente destructivo y deshumanizador, está destinado a desaparecer (Torres, 2019). Nuestro deber político y ético es buscar opciones viables y garantes de asegurar una vida digna para todos.

\section{REFERENCIAS BIBLIOGRÁFICAS}

Abu-Bakker, H. (2002). Eleven comments about otherness, equality and multiculturalism. Panim, 22, 32-38.

Aguado-Odina, T. (2017). El enfoque intercultural en educación. En T. Aguado-Odina, T. y P. Mata Benito (Coords.), Educación intercultural (pp. 17-46). UNED. 
Aguado-Odina, T., Mata-Benito, P., y Gil-Jaurena I. (2017). Mobilizing intercultural education for equity and social justice. Time to react against the intolerable: A proposal from Spain. Intercultural Education 28(4), 408-423. https://doi.org/10.1080/14675986.2017.1333874

Alim, H. S. \& Paris, D. (2017). What is culturally sustaining pedagogy and why does it matter? En D. Paris \& H. S. Alim (Eds.), Culturally sustaining pedagogies (pp. 1-21). Teachers College Press.

Au, W. (Ed.). (2009). Rethinking multicultural education: Teaching for racial and cultural justice. Rethinking Schools.

Augoustinos, M., Tuffin, K., \& Every, D. (2005). New racism, meritocracy and individualism: Constraining affirmative action in education. Discourse \& Society, 16(3), 315-340. https:// doi.org/10.1177/0957926505051168

Banks, J. A. (2004). Multicultural Education: Historical Development, Dimensions, and Practice. En J. A. Banks, \& C. A. M. Banks (Eds.), Handbook of Research on Multicultural Education (pp. 3-29). Jossey-Bass.

Banks, J. A. (2010) Multicultural education: Characteristics and goals. En J. A. Banks \& C. A. Banks (Eds.), Multicultural education: Issues and perspectives (pp. 3-30). Wiley.

Banks, J. A. (2013). The construction and historical development of multicultural education, 1962-2012. Theory into Practice, 52(1), 73-82. https://doi.org/10.1080/00405841.2013. 795444

Bartolomé Pina, M. (2004). Educación intercultural y ciudadanía. En J.A. Jordán Sierra, X. Besalú, M. Bartolomé Pina, T. Aguado-Odina, C. Moreno García y M. Sánz, La formación del profesorado en Educación Intercultural (pp. 93-122). Catarata.

Bhopala, K., \& Rhamie, J. (2014). Initial teacher training: understanding 'race,' diversity and inclusion. Race Ethnicity and Education, 17(3), 304-325. https://doi.org/10.1080/1361 3324.2013 .832920

Boler, M. (1997). The risks of empathy: Interrogating multiculturalism's gaze. Cultural Studies, 11(2), 253-273. https://doi.org/10.1080/09502389700490141

Caetano, A. P., Freire, I. P., \& Machado, E. B. (2020). Student Voice and Participation in Intercultural Education. Journal of New Approaches in Educational Research, 9(1), 57-73. https://doi.org/10.7821/naer.2020.1.458

Carbonell, J. (1995). Inmigración, diversidad cultural, desigualdad social y educación. Ministerio de Educación y Ciencia.

Cochran-Smith, M. (2010). Toward a theory of teacher education for social justice. En M. Fullan, A. Hargreaves, D. Hopkins \& A. Lieberman (Eds), The International Handbook of Educational Change (pp. 1-31). Springer Publishing.

Collins, P.H. \& Bilge, S. (2016). Intersectionality. Polity Press.

Commisision on Multicultural Education. (1973). No one model American: A statement on multicultural education. American Association of Colleges of Teacher Education.

Comisión Europea. (2017). Reforzar la identidad europea mediante la educación y la cultura. Comunicación de la Comisión al Parlamento Europeo, al Consejo, al Comité Económico y Social Europeo y al Comité de las Regiones. [COM(2017) 673 final]. https://eur-lex. europa.eu/legal-content/ES/TXT/?uri=CELEX:52017DC0673

Comisión Europea. (2018). Construyendo una Europa más fuerte: el papel de las políticas de juventud, educación y cultura. Comunicación de la Comisión al Parlamento Europeo, al 
Consejo, al Comité Económico y Social Europeo y al Comité de las Regiones. [COM(2018) 268 final]. https://eur-lex.europa.eu/legal-content/ES/TXT/PDF/?uri=CELEX:52018DC02 $68 \&$ from $=\mathrm{EN}$

Dedeoglu, H. \& Lamme, L. (2011). Selected demographics, attitudes, and beliefs about diversity of preservice teachers. Education and Urban Society, 43(4), 468-485. https://doi. org/10.1177/0013124510380718

European Commission. (2010). How can Teacher Education and Training policies prepare teachers to teach effectively in culturally diverse settings? Comisión Europea.

Essomba, M. (2010). Teacher education for diversity in Spain: moving from theory to practice. En OECD (Ed), Educating teachers for diversity. Meeting the challenge (pp. 219-236). OCDE/CERI

Ester Sánchez, A. T. (2017). La construcción de la identidad cultural desde una perspectiva de la educación multicultural. En R. Gonçalves y F. da Silva Veiga (Dirs.), Los desafíos jurídicos a la gobernanza global: una perspectiva para los próximos siglos (pp. 411-422). Advocacia-Geral da União.

Fiol-Mata, L. \& Chamberlain, M. K. (1994). Women of Color and the Multicultural Curriculum. The Feminist Press.

Freire, P. (1984). Pedagogía del Oprimido. Siglo XXI. 9. a edición.

Freire, P. (2001). Cartas a quien pretende enseñar. Siglo XXI.

Friant, N. et Sánchez-Santamaría, J. (2018). Conceptions de la justice en éducation: construction d'un cadre d'analyse. Teoría de la Educación, 30(1), 157-177. https://doi.org/10.14201/ teoredu301157177

Fukuyama. F. (2019). Identidad. La demanda de dignidad y las politicas de resentimiento. Deusto.

Garreta i Bochaca, J. (2004). El espejismo intercultural. La escuela de Cataluña ante la diversidad cultural. Revista de Educación, (333), 463-480.

Gay, G. (1995). Mirror images on common issues: Parallels between multicultural education and critical pedagogy. En C. E. Sleeter \& P. L. McLaren (Eds.), Multicultural education, critical pedagogy, and the politics of difference (155-189). State University of New York Press.

Giroux, H. A. (1990). Los profesores como intelectuales transformativos. Hacia una pedagogía crítica del aprendizaje. Paidós.

Giroux, H. A. (2019). La guerra del Neoliberalismo contra la educación superior. Herder.

Gorski, P. C. (2009). What we're teaching teachers: An analysis of multicultural teacher education coursework syllabi. Teaching and Teacher Education, 25(2), 309-318. https://doi. org/10.1016/j.tate.2008.07.008

Gramigna, A. (2007). Margine e método. Riflessioni su di una pedagogía della marginalità. Cuestiones pedagógicas, (18), 229-238.

Grant, C. \& Gibson, M. (2011). Diversity and teacher education. A historical perspective on research and policy. En A. Ball \& C. Tyson (Eds), Studying diversity in teacher education (pp. 19-62). AERA.

Hargreaves, A. y Shirley, D. (2012). La cuarta vía. El prometedor futuro del cambio educativo. Octaedro.

Harvey, D. (2007). Breve historia de neoliberalismo. Akal. 
VIRGINIA GUICHOT-REINA

EDUCACIÓN, JUSTICIA SOCIAL Y MULTICULTURALISMO: TEORÍA Y PRÁCTICA EN EL AULA

Herrera, S. G., Holmes, M. A., \& Kavimandan, S. K. (2012). Bringing theory to life: Strategies that make culturally responsive pedagogy a reality in diverse secondary classrooms. International Journal of Multicultural Education, 14(3), 1-19. https://doi.org/10.18251/ ijme.v14i3.608

Hinojosa Pareja E. y López López M. (2018). Interculturality and Teacher Education. A Study from Pre-service Teachers' Perspective. Australian Journal of Teacher Education, 43(3), 73-92. https://doi.org/10.14221/ajte.2018v43n3.5

Howard, T. C. (2003). Culturally relevant pedagogy: Ingredients for critical teacher reflection. Theory into Practice, 42(3), 195-202. https://doi.org/10.1207/s15430421tip4203_5

Hunter, C., Pearson, D. y Gutiérrez, R. (2015). Interculturalization and teacher education. Routledge.

Judt, T. (2012). Postguerra. Taurus. 7. a edición.

Kymlicka, W. (2013). Neoliberal multiculturalism? En P. A. Hall \& M. Lamont (Eds.), Social resilience in the neoliberal era (pp. 99-124). Cambridge University Press.

Lawyer, G. (2018). The Dangers of Separating Social Justice from Multicultural Education: Applications in Higher Education, International Journal of Multicultural Education, 20(1), 86-101. https://doi.org/10.18251/ijme.v20i1.1538

Leite, C. (2005). El currículum escolar y el ejercicio docente ante los desafíos de la multiculturalidad en Portugal. Profesorado, Revista de currículum y formación del profesorado, $9(2), 1-18$.

Leiva, J. J. (2008). Interculturalidad, gestión de la convivencia y diversidad cultural en la escuela: un estudio de las actitudes del profesorado. Revista Iberoamericana de Educación, 46(2), 1-14.

Milanovic, B. (2018). Desigualdad mundial: un nuevo enfoque para la era de la globalización. FCE. Económica.

Moloney, R., \& Saltmarsh, D. (2016). 'Knowing your students' in the culturally and linguistically diverse classroom. Australian Journal of Teacher Education, 41(4), 79-93. https:// doi.org/10.14221/ajte.2016v41n4.5

Muchenje, F. (2017). Cognitive justice and indigenous knowledge systems in the postcolonial classroom. En E. Shizha \& N. Makuyaza (Eds.), Re-thinking postcolonial education in Sub-Saharan Africa in the 21st century: Post-Millennium development goals (pp. 69-83). SensePublishers.

Nelson, S. W. \& Guerra, P. L. (2013). Educator beliefs and cultural knowledge. Implications for school improvement efforts. Educational Administration Quarterly, 50(1), 67-95. https://doi.org/10.1177/0013161X13488595

Nieto, S. \& Bode, P. (2012). Affirming diversity: The sociopolitical context of multicultural education. Pearson. $6^{\text {th }}$ ed.

Oakes, J., Lipton, M., Anderson, L., \& Stillman, J. (2016). Teaching to change the world. Routledge.4th ed.

OECD. (2009). Informe TALIS. La creación de entornos eficaces de enseñanza y aprendizaje. Santillana.

OECD. (2010). Reviews of Migrant Education. Closing the Gap for Immigrant Students: Policies, Practice and Performance. OECD. 
OECD. (2018). A Broken Social Elevator? How to Promote Social Mobility. OECD Publishing. Parhek, B. (2005). Repensando el multiculturalismo. Istmo.

Paris, D. (2012). Culturally Sustaining Pedagogy: A Needed Change in Stance, Terminology, and Practice, Educational Researcher, 41(3), 93-97. https://doi.org/10.3102/0013189X124412

Pinedo Castillo, I. A. (2013). Filosofía multicultural y educación para la convivencia ciudadana, Praxis \& Saber, 4(7), 179-199. https://doi.org/10.19053/22160159.2055

Puelles Benítez, M. (2010). Elementos de política de la educación. UNED.

Ruiz de Lobera, M. (2004). Metodología para la formación en educación intercultural. Catarata.

Salazar, M. C. y Ríos, F. (2016). Just scholarship! Publishing academic research with a social justice focus. Multicultural Perspectives, 18(1), 3-11. https://doi.org/10.1080/15210960 .2016 .1127073

Schön, D. A. (1987). Educating the reflective practitioner: Toward a new design for teaching and learning in the professions. Jossey Bass.

Shannon-Baker, P., \& Talbot, S. (2016). Even the dirt is dangerous: Racism in U.S. American study abroad programs. En V. Stead (Ed.), RIP Jim Crow: Fighting racism through higher education policy, curriculum, and cultural interventions (pp. 405-416). Peter Lang Publishing.

Shannon-Baker, P. (2018). A Multicultural Education Praxis: Integrating Past and Present, Living Theories, and Practice. International Journal of Multicultural Education, 2O(1), 48-66. https://doi.org/10.18251/ijme.v20i1.1518

Tarozzi, M. (2014). Building an «intercultural ethos» in teacher education. Intercultural Education, 25(2), 128-142. https://doi.org/10.1080/14675986.2014.888804

Torres López, J. (2019). España, hacia el caos sin remedio (como el resto del mundo). https://www.nuevatribuna.es/opinion/juan-torres-lopez/espana-caos-economia-crisisdesorden/20191109140525167949.html

Torres Santomé, J. (2008). Diversidad cultural y contenidos escolares. Revista de Educación, (345), 83-110.

Ukpokodu, O. N. (2016). Realizing transformative learning and social justice education: Unpacking teacher education practice. En S.M.T. Tomlinson-Clarke \& D. L. Clarke (Eds.), Social justice and transformative learning: Culture and identity in the United States and South Africa (pp. 113-142). Routledge.

UNESCO. (2006). UNESCO guidelines on intercultural education. UNESCO.

Yang, Y. \& Montgomery, D. (2011). Behind Cultural competence: the role of causal attribution in multicultural teacher education. Australian Journal of Teacher Education, 36(9), 1-21 https://doi.org/10.14221/ajte.2011v36n9.1 